\title{
Décrire les exotismes : quelques propositions
}

\section{Leonid Heller}

\section{(2) OpenEdition}

Journals

Édition électronique

URL : http://journals.openedition.org/edl/447

DOI : $10.4000 /$ edl. 447

ISSN : 2296-5084

\section{Éditeur}

Université de Lausanne

\section{Édition imprimée}

Date de publication : 15 septembre 2009

Pagination : 317-348

ISBN : 978-2-940331-20-8

ISSN : 0014-2026

\section{Référence électronique}

Leonid Heller, « Décrire les exotismes : quelques propositions », Études de lettres [En ligne], 2-3 | 2009, mis en ligne le 15 septembre 2012, consulté le 18 décembre 2020. URL : http://

journals.openedition.org/edl/447; DOI : https://doi.org/10.4000/edl.447 


\section{DÉCRIRE LES EXOTISMES : QUELQUES PROPOSITIONS}

Cet article tente d'ordonner la masse de définitions et d'interprétations de l'exotisme; il s'appuie sur une analyse lexicographique et sur des travaux français, particulièrement nombreux dans ce domaine. Après un parcours à travers la terminologie et les lectures modernistes et postmodernes du phénomène, nous répertorions les critères pouvant servir à classifier ses manifestations.

Là, tout n'est qu'ordre et beauté,

Luxe, calme et volupté.

Charles Baudelaire

Tout y ressemblait à une page, écrite dans une langue inconnue, entourée d'une arabesque.

Alexandre Grine

Sans vouloir épuiser la matière, nous aimerions esquisser dans cet article les contours de la notion d'« exotisme», afin de fournir un cadre systématique, sinon cohérent, à nos questions sur le fonctionnement de cette notion dans la culture russe. Sur le plan théorique, nous nous fonderons avant tout sur des travaux français, particulièrement nombreux et pertinents dans ce domaine; nous espérons ainsi donner une assise à une recherche comparée à venir.

\section{Terminologie de l'exotique}

La langue russe dispose, outre l'adjectif èkzotičeskij (avec sa variante ekzotičnyj et ses différents dérivés, tels l'adverbe èkzotično et le substantif 
èkzotičnost'), de deux substantifs que certains dictionnaires ${ }^{1}$ présentent comme des synonymes, èkzotizm et èkzotika. Pourtant, leur morphologie contredit l'idée de la synonymie; si le premier terme, calqué du français, garde la sémantique abstraite véhiculée par le suffixe-isme, le second, forme russifiée venue du latin via l'allemand (exoticus-exotica, ExotikumExotika), renvoie à une série d'objets concrets, aux choses exotiques. Ce couple lexical offre en russe (de même que ses homologues en allemand ou en polonais) la possibilité directe de marquer la différence entre un mode de penser ou un jugement (èkzotizm) et des objets et/ou événements présentant certaines caractéristiques (èkzotika). En remarquant au passage qu'on peut encore faire appel à èkzotičnost' pour généraliser ces caractéristiques, on dira que le syntagme «èkzotika africaine» recouvre les aspects de l'univers africain qui font que celui-ci est perçu comme «exotique». En revanche, l'«èkzotizm africain" renvoie à une fascination qui peut, en dehors du continent africain lui-même, susciter une mode pour certains tissus et ornements folkloriques, nourrir un nouveau genre de prose (le roman colonial), ou influencer l'esthétique d'un courant comme au début du XXe siècle, quand le cubisme a vécu une vraie passion pour l'«art nègre" (cette passion gagne vite la Russie où le théoricien de l'avant-garde letton Voldemars Matvejs consacre à l'art africain un livre important, le premier du genre en russe) ${ }^{2}$.

C'est en pensant à de tels cas que nous emploierons le terme «exotisme» (èkzotizm) pour signifier avant tout une orientation imprimée à l'acte créatif - qu'il s'agisse d'un poème, d'un traité philosophique ou d'un slogan publicitaire. Aussi allons-nous exclure de nos réflexions ce terme dans son sens linguistique de mot emprunté ${ }^{3}$, de même que toutes les fois où le langage courant lui attribue le sens de "bigarré», «inhabituel», «excentrique " ${ }^{4}$. Osons une définition quasi tautologique qui met en lumière non pas la sémantique mais les relations fonctionnelles des termes entre eux. On dira que l'acte de perception/description / (re)constitution du monde est marqué par l'exotisme lorsqu'il sert à exotiser son objet, c'est-à-dire à le doter de caractéristiques considérées

I. Par exemple, Slovar' sovremennogo ruskogo literaturnogo jazyka v 17 tt. (1965); E. Efremova, Novyj slovar' russkogo jazyka.

2. V. Markov, Iskusstvo negrov. Cf. Voldemar Matvej $i$ «Sojuz molodeži».

3. Voir dans ce recueil l'article d'Ekaterina Velmezova. Cf. aussi K. Bogdanov, O krokodilahv Rossii. Očerki iz istorii zaimstvovanij i èkzotizmov.

4. Cf., par exemple, Slovar' sovremennogo ruskogo literaturnogo jazyka v $17 \mathrm{tt}$. 
comme exotiques dans le milieu culturel au sein duquel l'acte en question est accompli. On reviendra sur les éléments qui composent cette définition. Pour l'instant, on y notera la position nodale du qualificatif "exotique»: les autres termes en dérivent; la lexicographie confirme la primauté de ce vocable.

Formé sur la racine exo ("au-dehors»), exotikos, étranger, semble être ignoré de la littérature grecque; on trouve en revanche exoticus chez Plaute. Bien plus tard, François Rabelais francise le mot en parlant de "diverses tapisseries, divers animaux, poissons, oiseaux et autres marchandises exoticques et pérégrines qui estoyent en l'allée du môle et par des halles du port" (Quart Livre, 1548). Ben Jonson parlera de «magick Witchcraft, or other such exotick arts" (Every Man in his Humor, 1599) ${ }^{5}$ - le sens ici se rapproche de «secret, esotérique». Malgré un début si prometteur, le mot sera utilisé de manière peu fréquente; il lui faudra plus d'un siècle et demi pour s'imposer. Le dictionnaire de Nathan Bailey (1756) resserre son sens («exoticalness: outlandishness; exotical: brought out of another country; exoticks: foreign plants»). Le Dictionnaire de l'Académie française (1762) confirme et ajoute, à côté des plantes, les "termes exotiques». Dans les dictionnaires allemands, différentes formes du mot apparaissent tôt, depuis le XVII ${ }^{\mathrm{e}}$ siècle, mais sans régularité ${ }^{6}$. Son entrée dans la langue russe est confirmée par le dictionnaire de Dahl (1864) : «Ekzotičeskij. Čuže(ino)zemnyj, iz žarkih stran; rast.» ${ }^{7}$

Aussi, pendant longtemps, le vocable a-t-il le sens assez restreint de "ce qui n'est pas du pays» et se réfère le plus souvent aux plantes. C'est vers le milieu du XIX ${ }^{\mathrm{e}}$ siècle qu'il va s'imprégner des nuances sémantiques que nous lui connaissons; grâce surtout à la littérature française, aux écrits de Gautier, Baudelaire, Hugo, Goncourt, le goût de l'exotique gagne l'Europe tout entière, mélange d'attirance pour le monde étranger et de volonté d'échapper au sien, moins naturel, moins beau, moins vivant car plus «embourgeoisé».

Quant au terme exotisme, s'il n'a peut-être pas été inventé en France (on trouve, par exemple, Exotismus dans l'échange épistolaire entre

5. F. Jost, «Exotisme».

6. Cf. H. Schulz, O. Basler, Deutsches Fremdwörterbuch. Mais le mot est absent de F. W. Looff, Allgemeines Fremdwörterbuch. En revanche le Fremdwörterbuch de Heyse (1807) introduit un couple destiné à disparaître: Exotikomanie-Exotikudenie pour traduire étrangomanie: cf. C. Rincón, "Exotisch/Exotismus».

7. "Exotique, étranger, venu des pays chauds; plantes". 
Goethe et le duc Charles Auguste) ${ }^{8}$, il est accueilli dans les salons parisiens au début des années 1860 - les frères Goncourt parlent du «sens de l'exotisme" en pensant déjà au "sentiment" et au goût de l'exotique ${ }^{9}-$ et deviendra courant vers la fin du siècle.

Enfin, si l'on en croit les dictionnaires, il faut attendre les années 1920 pour voir s'enraciner dans la langue russe le mot èkzotizm; d'après nos observations, on utilise alors bien plus fréquemment èkzotika, qui qualifie, avec une teinte plus ou moins marquée d'ironie, les attributs et les réalités des pays lointains. Ainsi, dans un roman satirique publié en 1931, qui relève avec beaucoup d'acuité les nouvelles habitudes langagières, l'apparition du premier chameau lors d'un voyage à travers les steppes kazakhes est commentée par: "L'Èkzotika commençait " (načalas' èkzotika) ${ }^{10}$.

A peine la mode exotique s'installe-t-elle que, déjà, Alphonse Daudet en dresse un bilan parodique dans son fameux Tartarin de Tarascon (1872). Don Quichotte petit-bourgeois des temps nouveaux, Tartarin baigne dans le bonheur au milieu de ses fantaisies. Son jardin est une collection de plantes exotiques (le mot d'ailleurs n'apparait dans le roman qu'à cet endroit), sa maison, une collection d'armes venues d'ailleurs, sa bibliothèque n'est composée que de livres de voyage et de chasse aux lions. L'imagination de Tartarin mélange tout, les Indiens Sioux, les grizzlis, les bandits des Abruzzes et les pirates malais, les Tatars, les Nègres et les Chinois. Dans le monde exotique, nous dit Daudet, c'est l'ambiance qui compte, les détails y sont entièrement subordonnés, ils sont interchangeables. Autre leçon tartarinesque: l'exotisme manie des clichés et conduit rapidement au kitsch.

Tout ce qui fait l'ambiance des récits de voyages, d'explorations, d'aventures - mystère, amour du danger et du risque, nostalgie de la pureté et de la beauté idéale, rêve de pouvoir et peut-être, avant tout, espoir d'une possibilité de transgression sans limites (songeons au Couur des ténèbres de Conrad) - tout cela intègre la nouvelle configuration sémantique de l'exotique. S'y ajoutent peu à peu d'autres nuances: énigmatique, fantasque, bizarre, excentrique, même glamour (pour les

8. Cf. H. Schulz, O. Basler, Deutsches Fremdwörterbuch.

9. E. et J. Goncourt, Journal. Mémoires de la vie littéraire, t. 5, p. 159, t. 6. p. 154, [20.2.1860, 23.8.1862, 23.11.1863].

Io. I. Il'f, E. Petrov, Zolotoj telenok, p. 169. 
Américains). Dans quelques domaines de la vie occidentale, l'exotique devient pratiquement équivalent de l'érotique: exotic dance et "striptease" sont synonymes (il faut dire que le fantasme d'une sexualité «naturelle», ludique et libre, est un ingrédient permanent des représentations exotiques). Ainsi, malgré l'âge respectable du mot dans sa forme première, son sens reste longtemps très restreint; ce n'est que vers la fin du XIX ${ }^{\mathrm{e}}$ siècle qu'il connaît une expansion fabuleuse, dynamisée par la tension entre ses pôles de "fuite» et de "curiosité désirante». S'établit alors une interaction: l'exotique se reconnaît grâce au "sens de l'exotisme» et celui-ci s'amplifie et évolue au fur et à mesure des découvertes et des reconnaissances de l'exotique. Toute cette dynamique agit à plein régime dans le champ sémantique et symbolique du modernisme. Nous en ressentons encore les effets, près de cent cinquante ans plus tard.

\section{Histoire des exotismes}

Nous venons d'établir que le sens actuel du mot "exotique» est relativement récent; or, certains chercheurs vont chercher loin dans le passé le phénomène complexe que le terme désigne, tel Tsvetan Todorov qui voit en Homère le premier "exotiste» ${ }^{11}$. On a parfois l'impression que toute relation d'un voyage lointain peut se lire sous l'angle de l'exotisme. Si l'on tient compte cependant de la composante "escapiste" de l'exotisme tel qu'il se manifeste au XIX ${ }^{\mathrm{e}}$ siècle, il faudrait conclure que les Grecs et les Romains ne devaient pas connaître de sentiment semblable: ils valorisaient trop leur univers pour sentir véritablement l'appel d'un ailleurs. Cela dit, ils connaissaient bien la curiosité de l'étranger et de l'étrange - autre aspect de l'exotisme. Cet Autre, dont la présence est toujours ressentie chez les Anciens, est à la fois uniformisé (tous les autres sont des Barbares) et multiple. Il est proche, méditerranéen: les Egyptiens, les Phéniciens; moins proche: les Perses, les Parthes; lointain: les Scythes, les Indiens; très lointain : les Hyperboréens. Ou encore ces peuples fabuleux qui habitent les marges du monde - Alexandre les a rencontrés, Pline l'Ancien les a décrits:

II. T. Todorov, Nous et les autres. La réflexion française sur la diversité humaine. Cf. aussi, G. Pochat, Der Exotismus während des Mittelalters und der Renaissance. Voraussetzungen, Entwiklung und Wandel eines bildnerischen Vokabulars. 
blemmyes, schiapodes, acéphales, cynocéphales, etc., autant d'images d'humanités possibles et inférieures dont notre imaginaire n'a pas fini de faire le tour. Les Anciens savaient s'étonner; leur attention est en permanence attirée par les merveilles (thaumata, mirabilia); mythes, légendes, expériences de voyages, de guerres, de négoce, extrapolations intellectuelles, tout concordait pour assurer qu'il existait des pays, des peuples, des formes de vie étonnants, qui méritaient la description. Parfois on allait jusqu'à adopter telle croyance, telle doctrine, tel savoir. Ces principes ne perdront plus leur validité. Les dragons gothiques emprunteront leur apparence aux dragons chinois ${ }^{12}$. Larabesque restera pendant des siècles le grand modèle d'ornement plastique et poétique. Les éléments génériques, iconographiques, topiques du récit de voyage et de rencontre avec l'extraordinaire servent encore aujourd'hui à la fabrication de l'Altérité et de l'Etrangeté. A l'époque des grandes découvertes et après, lorsque se jouera le grand drame colonial avec son accompagnement, l'épopée de la conquête du monde déroulant ses épisodes tropicaux, polaires, sous-marins, cosmiques, la matière de l'imaginaire gardera l'ancienne trame, entre l'attrait et le rejet, la curiosité et la peur, la volonté d'uniformiser l'Autre et la tentation d'affronter sa diversité.

N'oublions pas: l'exotisme n'est pas une qualité, c'est une relation qui dépend de nombreuses conditions parmi lesquelles figure en bonne place sa fonction pragmatique.

Peut-on qualifier d'exotiques les récits de voyages de Lucien ou de Swift? Les intentions satiriques, morales, parodiques et ludiques enfin ont plus d'importance dans ces récits que la découverte de l'inconnu. Pourtant, ils exploitent l'intérêt pour l'Autre tout en contribuant à le stimuler, même si ce n'est pas leur fonction dominante.

Un autre exemple: Pierre Vidal-Nacquet a analysé le mythe de l'Atlantide raconté par Platon ${ }^{13}$. Sa conclusion est que Platon ne raconte pas l'Atlantide, il élabore une allégorie de la décadence d'Athènes. Autrement dit, il utilise le cadre d'un récit de l'Autre pour faire un récit - distancié - de Soi. L'exotisme n'a que faire dans une pareille construction. Mais le mythe survivra jusqu’à nos jours en sa qualité de

I2. Cf. J. Baltrusaitis, Le Moyen Age fantastique. Antiquités et exotismes dans l'art gothique.

13. P. Vidal-Nacquet, Le Chasseur noir. Formes de pensée et formes de société dans le monde grec. 
mythe d'une part, en sa qualité de cadre pour la description de mondes exotiques, d'autre part.

La découverte de l'Amérique et le siècle des Lumières et des voyages autour du monde constituent deux moments essentiels pour l'histoire de l'exotisme, ou plutôt des exotismes; mais ni l'une ni l'autre de ces époques n'a associé son attitude envers les mondes nouveaux au terme exotique, pourtant déjà connu. François Hartog a étudié ces deux moments en suivant les discussions qui se déchaînent lorsque l'Europe étonnée découvre les "sauvages»: les Indiens d'Amérique, les Polynésiens, les Africains. Ils deviennent un écran sur lequel on fait la projection de rêves et de visions du monde. Des questions fusent. Sontils descendants d'Adam, c'est-à-dire humains, ou bien font-ils partie de l'ordre animal? Figurent-ils «l'enfance de l'humanité» en continuant à vivre de la façon dont vivaient les anciens Européens? Ne représententils pas la meilleure part de l'humanité, celle qui a évité la décadence par la civilisation et, dans ce cas, ne sont-ils pas un exemple à suivre pour l'humanité à venir? Les débats autour de ces questions déterminent les positions dans la querelle séculaire entre les Anciens et les Modernes et, partant, beaucoup d'aspects essentiels de la pensée européenne. Ces débats donnent naissance, au XVIII siècle, aux conceptions de l'«homme de la nature» et du "noble sauvage», à la compréhension du temps par analogie avec l'espace. Ils jettent les bases de l'exotisme du XIX ${ }^{\mathrm{e}}$ siècle ${ }^{14}$. Mais l'Encyclopédie de Diderot et d'Alembert ne consacre au mot «exotique» qu'une ligne en tant que terme de jardinage.

\section{Théorie de l'exotique: le modernisme}

On peut considérer l'exotisme comme une voie que le dernier tiers du XIXe siècle artistique emprunte pour s'échapper du réalisme. Le premier à adopter ce point de vue est, semble-t-il, le philosophe JeanMarie Guyau, auteur de L'Art au point de vue sociologique (1887) ${ }^{15}$, un grand succès international. Les tableaux de Delacroix, les récits des pays lointains depuis Bernardin de Saint-Pierre jusqu'à Pierre Loti remplissent selon Guyau deux fonctions; ils permettent de fuir la

I4. F. Hartog, Anciens, modernes, sauvages.

I5. J.-M. Guyau, L'Art au point de vue sociologique. 
trivialité de tous les jours (fonction sociale) et de rendre le monde poétique (fonction esthétique). Le trait fondamental de l'exotisme serait le pittoresque grâce auquel le regard sur le monde peut se renouveler.

$\mathrm{Au}$ début du $\mathrm{XX}^{\mathrm{e}}$ siècle, plusieurs critiques littéraires se réfèrent à Guyau pour faire de l'exotisme un objet d'études académiques ${ }^{16}$; ils soulignent les conséquences esthétiques de la passion pour l'exotique. Combiner l'onirisme motivé par la perception de l'inconnu avec un art de capter ce dernier au travers de détails concrets et sensuels: telle est la démarche exotique et c'est là que réside la garantie de sa modernité et son importance dans le combat pour le renouvellement de la convention réaliste ${ }^{17}$.

En pleine époque des avant-gardes, le critique de renom Jacques Rivière (Le Roman d'aventure, 1913) appelle les écrivains à abandonner les descriptions statiques des états d'âme et de la vie quotidienne pour apporter à la littérature une tension et un rythme accélérés ${ }^{18}$. Sans refuser la psychologie, Rivière demande de raconter les événements de la pensée et de l'esprit comme autant d'aventures et d'utiliser pour cela une convention narrative adéquate; il propose de trouver le monde exotique au fond de soi-même.

Enfin, entre 1904 et 1918, le poète et voyageur Victor Segalen multiplie les notes en vue d'une grande étude sur l'exotisme. Ces notes ne seront réunies et éditées qu'en $1978{ }^{19}$, au moment de la sortie du livre de Said (la coïncidence vaut d'être notée) et attireront l'attention générale sur le sujet. Les opinions de Segalen, auteur d'un roman exotique très populaire Les Immémoriaux (1907) et d'écrits sur Gauguin (après la mort du peintre, il a retrouvé et sauvé à Tahiti quelquesuns de ses tableaux parmi les plus célèbres), étaient connues de son vivant; après la sortie du livre, elles vont influencer toutes les théories de l'exotisme d'aujourd'hui. Ses formules sont étonnantes de justesse et de précision. Il prédit notamment l'arrivée d'un tourisme massif et globalisé, funeste pour l'exotique. En analysant différents observateurs des mondes inconnus, il en définit trois types: le touriste qui consomme

I6. Cf. dans la bibliographie de cet article les ouvrages de Martino, Cassagne, Cario, Régismanset et Chinard.

17. Cf. G. Ducrey, J.-M. Moura (dir.), Crise fin-de-siècle et tentation de l'exotisme; A. Décoret-Ahiha, Les Danses exotiques en France. 1880-1940, etc.

I8. J. Rivière, Le Roman d'aventure.

19. V. Segalen, Essai sur l'exotisme, une esthétique du divers. 
des produits exotiques, le folkloriste qui étudie et tente de comprendre et d'expliquer l'exotique, enfin, le voyageur-exote ${ }^{20}$. Ce dernier collectionne la diversité, son attitude envers l'exotique est attentive et précautionneuse, il reconnaît à l'exotique l'unicité du sujet et évite de le réduire à l'accessible et au compréhensible. Segalen semble annoncer la pensée de Lévinas: "si tout est déductible, il n'y a pas l'Autre " ${ }^{21}$.

Ainsi, sous la plume de plusieurs praticiens et théoriciens français prend corps une vision de l'exotisme assez complète et cohérente. Nous pensons qu'elle n'a pas encore perdu sa validité. Un exemple: le sous-titre du livre de Tsvetan Todorov Nous et les autres, qui évoque la «réflexion sur la diversité» ${ }^{22}$, est un renvoi explicite à Segalen. Tentons de reconstruire la théorie moderniste de l'exotisme à partir des ouvrages évoqués.

1. L'attirance pour l'exotique est escapiste, elle vient de l'envie d'échapper au spleen, à la monotonie et au côté machinal de la vie moderne, urbaine, bourgeoise. Quelques années après Segalen, un théoricien allemand, en parlant de l'exotisme des sens, assimilera le goût de l'exotique à un besoin psychophysiologique; le plaisir procuré par l'exotisme se change en quête de sensations de plus en plus fortes ${ }^{23}$.

2. En même temps, la quête de l'exotique peut être qualifiée d'activisme esthétique; c'est une lutte contre la vision stéréotypée du monde et pour sa poétisation. Prise dans ce sens, l'exotisation apparaît comme un puissant procédé de la distanciation poétique. Que l'on nous pardonne de citer cet extrait mainte fois cité, tiré du journal de Gide, dans lequel se trouvent reformulées les opinions de Guyau:

Ce qui fait le charme et l'attrait de l'Ailleurs, de ce que nous appelons exotisme, ce n'est point tant que la nature y soit plus belle, mais que tout nous y paraît neuf, nous surprend et se présente à notre œil dans une sorte de virginité ${ }^{24}$.

20. Parfois, surtout en allemand, «exote» signifie habitant (ou produit) de pays exotique; à ne pas confondre avec l'emploi, inverse, qu'en fait Segalen.

2I. E. Lévinas, "Visage et violence première (Phénoménologie de l'éthique). Interview», p. 140.

22. T. Todorov, Nous et les autres. La Réflexion française sur la diversité humaine.

23. F. Brie, Exotismus der Sinne. Eine Studie zur Psychologie der Romantik. Cf.

C. Rincón, «Exotisch/Exotismus».

24. A. Gide, Journal. 1889-1939, p. 1236 (2.8.1935). Cf. la citation du même extrait dans l'article de Mme Coldefy-Faucard, p. 219. 
Cette réflexion aurait très bien pu apparaître, vingt ans plus tôt, sous la plume d'un Viktor Chklovski, mais au terme "exotisme» se serait substitué celui d' "ostranenie" (défamiliarisation), procédé de base de la représentation artistique selon les formalistes russes. Nous comptons explorer dans une autre étude ce parallèle entre les théories de l'exotisme et les conceptions formalistes.

3. L'exotisation "défamiliarisante" peut se manifester dans la perception du quotidien. Commentant un passage de Maupassant qui déclare que "la moindre chose contient un peu d'inconnu", Jean-Marc Moura convient: "Il est sans doute possible à l'œil subtil de discerner l'étrangeté au cœur du plus familier, et au bon écrivain de rendre "exotique" un paysage connu.» Mais d'ajouter aussitôt: "On conservera néanmoins la distinction entre l'exotisme, tourné vers les mondes non-européens, et le pittoresque, qui caractérise aussi bien l'évocation de contrées familières ${ }^{25}$. Selon nous, la distinction entre l'exotique et le pittoresque doit être maintenue pour une autre raison: ils entrent dans des catégories de niveaux différents, le premier concerne l'objet dans son être, le second, la manière de décrire l'objet. Ils ne s'excluent pas l'un l'autre, au contraire (nous avons vu la définition de Guyau). Par ailleurs, on connaît dans la littérature russe, depuis les récits ukrainiens de Gogol jusqu'aux récits d'Odessa d'Isaac Babel, la pratique de l'exotisation qui transforme le milieu quotidien du sujet-observateur en un Ailleurs fait d'émerveillement. Et l'on connaît, dans les romans d'Andreï Biély ${ }^{26}$, la représentation de la vie intérieure du narrateur sous la forme de paysages préhistoriques et sauvages, peuplés de personnages métamorphosés en animaux et en figures mythiques, fourmillant de dangers et de rencontres insolites. C'est à croire que Biély suit la recommandation de Rivière dans sa démarche: l'aventure la plus incroyable serait encore celle que l'enfant vit à l'intérieur de lui-même. En tout cas, Biély et Babel justifient à eux seuls que l'on introduise dans la discussion la notion d'auto-exotisation.

4. L'exotique n'existe pas en dehors de la perception de l'observateur. Il dépend non seulement de l'état affectif, de la sensibilité ou du sens de l'observation de ce dernier, mais tout autant des habitudes intellectuelles et culturelles qui forment les outils lui servant à affronter la nouveauté.

25. J.-M. Moura, Lire l'exotisme, p. 11-12.

26. Surtout Kotik Letaev (1915-1922). Cf. A. Biély, Kotik Letaïev. 
Ainsi, les Indiens d'Amérique sont d'abord perçus non comme des êtres vivants mais comme des schémas qui confirment ou infirment des théories déjà existantes ${ }^{27}$.

5. Inversement, le passage de l'observateur transforme le monde observé: ce dernier se modifie afin d'accueillir le voyageur et il garde longtemps, sinon toujours, des traces de ce passage - cette thèse de Segalen, la base de son éthique de l'exote, est devenue le postulat de départ de toute approche ethnologique et anthropologique aujourd'hui.

6. Todorov indique le paradoxe logique de l'exotisme: l'exotique est valorisé sans être connu, mais il est impossible d'attribuer une valeur à ce que l'on ne connaît pas $^{28}$. Nous ne voyons pas ici de difficulté insoluble. D'abord, on le sait au moins depuis Guyau, l'exotisme fonctionne selon les règles du désir et du fantasme. Ensuite, l'exotisation, en tant que projection, cache son objet autant qu'elle le montre (la pratique plasticienne de projeter des images vidéo sur le décor urbain illustre bien cette ambivalence) : dans ce jeu, qui est plein de sens pour l'exote, mais non pour le folkloriste ou l'ethnologue, il s'agit de préserver l'altérité dans son mystère en valorisant précisément ce mystère: on peut y voir une sorte de principe éthique d'uécologie de la diversité». Enfin - nous allons y revenir - les représentations de l'Ailleurs sont souvent liées, et ce depuis l'Antiquité, à l'idée du bonheur. Il n'y a donc rien d'étonnant à ce que l'Ailleurs soit valorisé par anticipation.

7. L'exotisation souligne la variété du monde, sa nouveauté inépuisable, le caractère unique de ses éléments, et donc la réalité très concrète de ses recoins les plus secrets. Or voilà un autre paradoxe (ou est-ce le même, mais formulé différemment?) : l'apparition de l'exotique force à s'interroger sur la cohérence du réel; l'observateur se retrouvant souvent dans un état quasi hypnotique/ narcotique/onirique, sa confiance dans la solidité du monde tel qu'il le connaît s'ébranle. C'est ainsi qu'Alexandre Grine, l'« exote» le plus accompli de la littérature du modernisme russe, peut écrire à propos de l'un de ses célèbres et énigmatiques personnages féminins: «Sa robe et sa silhouette respiraient la destruction de la réalité ${ }^{29}$.

27. Cf. C. Rincón, «Exotisch/Exotismus», p. 344.

28. T. Todorov, Nous et les autres. La Réflexion française sur la diversité humaine, p. 356.

29. «Ot plat'ja i figury ee vejalo razrušeniem dejstvitel'nosti». A. Grin, «Klubnyj arap ", p. 410. 
8. L'exotisme vise l'espace géographique associé aux pays lointains; lorsque la planète n'offre plus beaucoup d'endroits secrets, il gagne les dimensions temporelles, passé et futur. Segalen en parle notamment en prévision de la littérature d'aventures intersidérales. Tandis que l'exemple parfait d'une incursion dans le passé exotique est donné par Salammbô de Flaubert, livre culte pour tout le modernisme.

9. L'exotique littéraire se rattache en même temps à la tradition du roman de voyage et à celles de l'utopie et du récit bucolique (Diderot, Bernardin de Saint-Pierre, Rousseau le prouvent bien), du conte féérique et du fantastique. Ce n'est pas un hasard si la passion pour l'Orient exotique est lancée dans le monde des lettres européen par l'engouement pour les Contes des Mille et une nuits de Galland (1701), si la vague du fantastique gothique est annoncée par Vathek (1789) de Bedford, histoire exotique s'il en est.

10. Abstraction faite du domaine de l'art et du matériau choisi par l'exotisme pour se matérialiser, on dira que les ressorts poétiques les plus généraux de celui-ci sont l'ornementalisation (l'exploitation du pittoresque, souvent alliée à l'onirisme descriptif, en même temps que l'attention accordée au plan de l'expression) et l'extrême narrativisation (sur le mode du roman d'aventures) qui stimulent avant tout la perception sensuelle, affective et intuitive. Nous y ajouterons l'allégorisation, dispositif qui sert à relier la nouveauté de l'exotique à des réseaux sémiotiques et symboliques déjà opérants.

Le grand avantage de la conception que nous avons déduite de nos lectures modernistes est qu'elle traite l'exotisme comme une catégorie esthétique, sans minimiser ses aspects sociaux, idéologiques, etc. Il faudrait la compléter: la littérature anglo-saxonne est tout autant, sinon plus que la française, imprégnée d'exotisme; le romantisme allemand devrait pouvoir y apporter sa voix (Novalis situait en Atlantide le pays de la poésie) ${ }^{30}$. Mais ne nous dispersons pas, d'autant que certains critiques estiment, faisant référence à Montaigne, Montesquieu, Diderot, Flaubert, Loti, Segalen, que la théorie de l'exotisme est une invention française ${ }^{31}$. Il est certain en tout cas que les Russes, qui ont

30. N. Fernandez-Bravo, "Le Mythe de l'Atlantide dans le romantisme allemand", p. 210.

3I. Cf. R. Célestin, From Canibals to Radicals: Figures and Limits of Exoticism. 
lu Guyau ${ }^{32}$, qui ont suivi les débats autour de Gauguin provoqués par la sortie de Noa-Noa $(1901)^{33}$, connaissent cette tradition française et dialoguent avec elle.

\section{Théorie de l'exotique: le postmodernisme}

"Je hais les voyages et les explorateurs»: tel est le début de Tristes Tropiques (1955), célèbre livre de Claude Lévi-Strauss. L'anthropologue y qualifie de "scories de la mémoire» ses souvenirs d'aventures et accuse les fournisseurs et les consommateurs d'exotique de n'aimer que les effets superficiels et de manquer d'intérêt pour ce qui est le plus important dans les autres mondes, ces ordres profonds qui les organisent. On peut penser que c'est à partir de ce moment que commence la réévaluation de l'exotisme. Autant le modernisme en fut friand, autant le postmodernisme le rejette. Bientôt, Roland Barthes va déclarer que l'exotisme, ce mythe bourgeois par excellence, est vide d'imagination, ramollissant par son action et, donc, nocif ${ }^{34}$. Le délit d'exotisme entachera tout l'humanisme du XIX siècle que Lévi-Strauss appelle l'«humanisme exotique ${ }^{35}$, coupable d'européocentrisme, de collusion avec le colonialisme, de diffusion du racisme ${ }^{36}$.

Accusations lourdes et politiquement très correctes: une raison de s'en méfier. Les liens entre l'exotisme et le colonialisme sont considérés comme si évidents que toute analyse en devient superflue. Il serait absurde de nier de tels liens, il est bien facile de trouver des clichés racistes dans un roman colonial, de voir comment l'exotisme suit les vecteurs de l'expansion coloniale. Jean-Marc Moura écrit:

Chaque œuvre exotique doit être interprétée selon une triple détermination [...]. Comme produit d'une conscience créatrice rêvant l'étranger, comme forme exprimant la sensibilité exotique d'une

32. Le livre de Guyau est traduit en russe très tôt et plusieurs fois réédité:

Ž.-M. Gjujo, Iskusstvo s točki zrenija sociologii.

33. Cf. la préface de Ja. Tugendhol'd à Pol' Gogen, Noa-Noa. Putešestvie na Taiti.

34. R. Barthes, Mythologies.

35. C. Lévi-Strauss, "Trois humanismes», p. 322.

36. Cf. S. Bessis, L'Occident et les autres. Histoire d'une suprématie. 
époque, comme signe - plus ou moins manifeste - de l'histoire coloniale européenne ${ }^{37}$.

Ce qui revient à dire que la relation au colonialisme épuise le lien de l'œuvre avec son univers historique et culturel. Cette attitude du "colonisateur contrit» simplifie les choses. La fonction exotique ne fait pas qu'accompagner la démarche colonialiste. La colonisation n'est pas que l'extension de la "domination blanche», c'est aussi le corollaire de toute sédentarisation ou de toute "conquête de la Nature».

Conséquence de la même attitude, certains critiques affirment que la notion d'exotisme n'existe que dans le discours européen: Marco Polo a bouleversé la culture européenne, mais a été oublié en Chine. «Le cerisier japonais fleurit dans les littératures de l'Ouest, mais le sapin des Alpes n'a guère de place dans les lettres nippones " ${ }^{38}$. De telles affirmations révèlent des présupposés erronés, ceux de la parité et de la réciprocité simultanée dans les rapports entre les cultures. Elles sont invalidées, nous semble-t-il, par certains romans japonais (par exemple ceux de Haruki Murakami), dans lesquels les américanismes jouent le rôle de renvoi à un ailleurs attirant et méconnu, ou encore par la passion japonaise pour la littérature russe, ainsi que d'une manière plus générale, pour la culture européenne et, surtout, pour la musique classique: ne s'agit-il pas là d'un véritable "exotisme des sens"? D'autre part, après la victoire japonaise sur la Russie en 1905, un orientalisme particulier voit le jour dans les pays arabes - en réponse à celui des Occidentaux - qui incite à voir le Japon comme un pays oriental idéal ${ }^{39}$. Notons encore, dans le désordre, que dans un colloque sur l'exotisme organisé sur l'île de la Réunion, l'«exotique à rebours" a été abordé, lors de la présentation d'un récit de voyage de deux Africains en Europe ${ }^{40}$.

Bref, si nos connaissances sont trop lacunaires pour émettre des jugements arrêtés, nous soupçonnons l'existence dans d'autres cultures, sous des noms différents, de phénomènes proches de l'exotisme, comme il en existe de semblables dans le domaine de la xénophobie. La question

37. J.-M. Moura, Lire l'exotisme, p. 18.

38. F. Jost, «Exotisme».

39. A. Roussillon, Identité et modernité. Les voyageurs égyptiens au Japon (XIX $-X X^{e}$ siècle). Cf. aussi M. Abaza, "Japan as imagined by Arabs".

40. W. Zimmer, "Voyages africains de recherche et de découverte à l'intérieur de l'Allemagne. L'exotisme à rebours ". 
est importante car elle concerne la nature du lien entre l'exotisme et le colonialisme européen. Elle a son importance pour les études russes.

Nous ne pouvons dans cet article aborder la spécificité de la position russe face au colonialisme ${ }^{41}$. Bornons-nous à noter que le discours soviétique officiel fustige l'exotisme ${ }^{42}$ - proche en cela du discours postmoderne - comme une attitude bourgeoise, colonialiste et raciste. Cependant, la création du type orientaliste (au sens large) a une place importante dans la culture soviétique, aussi bien dans les années vingt et à l'époque stalinienne qu'après le Dégel; certains "romans de production" situés en Asie font office de véritables romans d'aventures coloniales, tel le roman de Bruno Jasienski L'Homme change de peau ${ }^{43}$; Andreï Platonov en fournit un anti-modèle avec Djann ( $D \check{z} a n$, vers 1935, publié en URSS trente ans plus tard). Plus tard, le schéma exotique fonctionne aussi bien dans des livres faisant partie du canon officiel (comme les romans de Tchinguiz Aïtmatov) que dans des œuvres anticonformistes telles que Stalker, le film d'Andreï Tarkovski ou, aujourd'hui, les romans "déconstructionnistes" d'un Pélevine ou d'un Sorokine. Il n'existe pas encore de travaux qui passeraient en revue et ordonneraient toute cette production.

La question que nous avons soulevée, celle du lien entre l'exotisme et le colonialisme, n'est pas traitée de manière convaincante dans les études existantes. Celles-ci montrent comment les Russes voient les objets exotiques (le Caucase, l'Asie centrale) ${ }^{44}$; et il va de soi qu'elles trouvent dans ce regard le reflet d'une volonté impériale de domination. L'inverse est peu connu en dehors d'évidences: le regard des membres de la «famille des peuples» tsariste ou soviétique sur leur Grand Frère n'a pas été abordé en termes d'exotisme et d'exotisation. Or, on doit d'abord chercher dans les images stéréotypées de la Russie produites par les littératures socialistes des «minorités nationales» les fissures qui révèlent la «vraie» perception du Russe dominateur - en faisant

4I. Quelques mots en ont été dits dans l'avant-propos.

42. Voir dans ce recueil l'article de Marietta Tchoudakova.

43. B. Jasenskij, Čelovek menjaet kožu.

44. Par exemple S. Layton, Russian Literature and Empire. Conquest of the Caucasus from Pushkin to Tolstoy; E. Thompson, Imperial Knowledge. Russian Literature and Colonialism; D. Schimmelpenninck van der Oye, Toward the rising sun: Russian ideologies of empire and the path to war with Japan. 
écho aux travaux postcoloniaux de Homi K. Bhabha, Gayatri Spivak et d'autres - et, ensuite, d'éventuelles traces d'un "exotisme inversé », d'une représentation de la Russie comme altérité lointaine et étrange. L'« exotologie» a beaucoup à faire dans le domaine russe.

Notons encore un problème en rapport avec ce qui vient d'être dit. L'exotisme revient dans le contexte postmoderne à l'occasion de la discussion sur l'Altérité. On admettra, à la suite de Foucault et Said, que l'exotisme et l'exotique, la représentation de l'Autre comme action et comme résultat d'action, sont non pas une donnée objective mais une construction. Sa première fonction est de consolider l'identité symbolique du sujet culturel/social, mais cette construction est exploitée à des fins pratiques - idéologiques, politiques, etc. Parfois, l'altérité ne se conçoit que comme un matériau pour l'édification de l'identité; parmi d'autres, Julia Kristeva soutient, avec la bénédiction (usurpée?) de Freud, que «L'autre c'est mon (propre) inconscient», que «L'autre est en nous " ${ }^{45}$. Une telle approche annule jusqu'à la possibilité de l'autonomie de l'Autre. De même, on se méfiera du binarisme Identité / Altérité qui semble bien simplifier le tableau. Prenons un exemple pour éclairer notre propos. François Hartog montre comment Hérodote, qui manie en permanence l'opposition Grecs-Barbares, la transpose pour traiter de l'affrontement Perses-Scythes; dans son modèle binaire, les Perses vont rester Barbares mais les Scythes vont reprendre le rôle des Grecs, avec toutes les incohérences que cette opération implique, notamment lors de l'évocation de la tactique militaire ou de l'armement. Ces incohérences ne peuvent être comprises qu'après une rectification des procédés d'inversion et de substitution ${ }^{46}$. De tels mécanismes rhétoriques peuventils suffire à rendre compte de la représentation moderne et complexe de l'exotisme? Nous préférons adhérer à la vieille théorie française et suivre Segalen pour distinguer, face au Soi, altérité, diversité et étrangeté (opaque, du moins partiellement, à notre intelligence), toutes ces nuances faisant partie de l'exotique.

45. J. Kristeva, Etrangers à nous-mêmes, p. 271, 283.

46. F. Hartog, Le Miroir d'Hérodote. 


\section{Classification des exotismes}

Abordons la classification des différentes formes de l'exotisme à travers les usages répertoriés par la lexicographie. Nous avons travaillé de manière systématique avec les dictionnaires et encyclopédies de langue russe, mais des ouvrages en d'autres langues ont également été mis à contribution. Nous présentons nos observations en les confrontant à ce que nous savons déjà de la théorie de l'exotisme, sous la forme d'une liste des mécanismes de la sémantisation / sémiotisation:

a) Mise à distance (y compris temporelle) : priment ici depuis toujours les notions de lointain, d'outre-mer, de déplacement spatial (voyage) comme forme de contact privilégiée avec l'exotique; ajoutons le rôle possible de l'itinéraire (rectiligne, circulaire, erratique...).

b) Mise en absence: l'idée de "non-être ici" et d' "être ailleurs"; la littérature exotique est "écriture de l'absence " ${ }^{47}$ ou de "la rareté", complétée par l'imagination. D'où la porte ouverte au féerique, au fantastique.

c) Mise en étrangeté: souvent, l'étrange est vu comme incompatible avec Soi ou avec la Nature; ce mécanisme fait s'activer nos représentations de la Norme et du Naturel (si nous pensons notre monde comme artificiel, l'exotisme cherchera le naturel ailleurs, etc.).

d) Défamiliarisation par l'extraordinaire (complément du point précédent) : notre étonnement implique la non-reconnaissance du différent et présuppose la connaissance de Soi et du Sien (de l'habituel) : cette dynamique de l'«épistémologie comparée» est essentielle dans la chasse à l'exotique (aussi bien que pour l'ostranenie, la distanciation formaliste).

e) Extériorisation de l'Autre, sa réduction à ses manifestations extérieures: c'est la position détachée, externe et mobile de l'observateur qui est responsable de cette situation, motivant relativismes, subjectivismes, constructivismes. Bien plus, on a pu écrire:

L'Occidental définit l'exotisme comme un appel aux saveurs et aux sens, l'excluant ainsi du domaine de la pensée à laquelle sa civilisation accorde une primauté certaine ${ }^{48}$.

47. J.-M. Moura, Lire l'exotisme, p. 133.

48. J.-P. Hassoun, A. Raulin, "Homo exoticus», p. 120. 
Cette remarque est vraie dans le sens où la grammaire indienne, la médecine chinoise ou les mathématiques arabes, qui existent bel et bien, ne sont pas forcément qualifiées d'"exotiques». On évite de qualifier ainsi une pensée ou un savoir car on a le réflexe de mettre l'universel au-dessus du local, réflexe qui ressemble à, mais ne se confond pas avec l'européocentrisme. Ainsi l'extériorisation ne résulte pas seulement de la dépréciation de l'Autre lointain. Elle a également son origine dans les conditions toutes pratiques de la perception. Pour nous, il est tentant de penser que l'extériorisation correspond à une représentation du monde revenue à son cadre épique, une sorte d'archaïsation du monde exotique selon les codes homériques qui ne connaissaient pas les profondeurs de l'intériorité ${ }^{49}$.

Les mécanismes dont nous avons dressé une liste non-exhaustive agissent de manière simultanée et complémentaire; la priorité de l'un ou de l'autre ainsi que leur poids respectif peuvent fonder une typologie de l'exotique interagissant avec la grille de conventions thématiques et génériques.

La première base de la classification est géographique (point [a] de la liste) : peuvent s'y combiner les critères d'éloignement et d'isolement, de climat, de paysage. On a l'habitude d'associer l'exotisme aux régions chaudes (le dictionnaire de Dahl le note explicitement): la chose est entendue, c'est de là que venaient les marchandises exotiques. Mais la recherche du passage du Nord-Ouest, la course aux pôles Nord et Sud, la fièvre de l'or en Alaska ont transformé la donne. Il est significatif que dans l'œuvre d'un Jack London les récits des mers chaudes répondent en écho aux histoires du Grand-Nord. La conquête de l'Arctique trouvera des représentations différentes de celles de l'Afrique, mais non moins spectaculaires ni moins essentielles, surtout pour l'imaginaire russe ${ }^{50}$. Cette série peut être complétée par une série «chronographique» ( $L a$ Machine à remonter le temps de $\mathrm{H}$. G. Wells montre comment elles peuvent se combiner).

49. Sur cette question de l'«extériorité» plastique grecque, cf. E. Auerbach, Mimésis. La représentation de la réalité dans la littérature occidentale; J.-P. Vernant, Entre mythe et politique, etc.

50. Voir dans ce volume l'article d'Anne Coldefy-Faucard. 
Dans une typologie fondée sur les valeurs (points [b], [c], [d]), le naturel s'opposerait à l'artificiel, la simplicité à la luxuriance, le raisonnable au passionnel.

D'autres classifications mettraient en lumière les aspects épistémologiques, les modes de perception de l'exotique, plus ou moins imaginaire ou réaliste, sensualiste ou rationnaliste, critique ou utopique (points [b], [c], [d]). La topologie de la perception devrait être prise en compte, qui dépend du positionnement et de la nature de l'observateur en même temps que de l'organisation de l'espace de l'observation, des directions possibles du regard et de la projection (points [c], [d], [e]). L'histoire du Scythe Anacharsis, venu à Athènes pour se cultiver et tué à son retour dans sa patrie pour avoir tenté de "gréciser" les mœurs, histoire racontée par Hérodote et Lucien, illustre bien la structure anisothrope, organisée différemment selon la direction que l'on prend, de cet espace de perception. Reconnaître la structure en question est primordial pour qui étudie la culture russe et surtout soviétique; plus généralement, c'est important pour suivre les flèches d'exotisation. On connaît bien l'inversion exotisante, comme dans les Lettres persanes de Montesquieu. Nous pouvons parler de rétro-exotisation (la mise à distance de l'époque soviétique sert à en fabriquer aujourd'hui des images nostalgiques pouvant satisfaire un goût proche de l'exotisme) ${ }^{51}$. Nous avons évoqué plus haut l'auto-exotisation d'un Biély. Le célèbre poème d'Alexandre Blok, «Les Scythes» (1918), présente la Russie en révolution comme une force brutale lancée depuis l'espace et le passé asiatiques pour «briser l'échine» d'une Europe dégénérée. Le poème peut être lu comme une tentative d'auto-barbarisation, l'élaboration d'un «Soi exotique" adressée à un Autre construit, lui, à la fois comme observateur et comme instance centrale, normalisatrice. D'autres formes d'inversions sont possibles. Sergueï Essénine, en visite aux USA, se surprend à penser à ses compatriotes paysans comme à de "pauvres Hiawatha russes». Alexandre Etkind attire très justement l'attention sur le processus de "colonisation intérieure" en Russie et sur le fait que les études sur la paysannerie russe sont organisées au XIX siècle selon le modèle

5I. Natal'ja Samutina l'a évoqué dans sa communication au colloque Exotismes dans la culture russe, non incluse dans ce volume; cf. son article "Kul'tovoe kino: daže zritel' imeet pravo na svobodu». 
des expéditions dans des pays exotiques ${ }^{52}$. On peut seulement ajouter qu'à d'autres échelles, ce phénomène est connu un peu partout et que les régions gardent jusqu'à nos jours un certain "parfum exotique", comme en témoigne le succès en France de livres comme Le Cheval d'orgueil de Pierre Jaquez Hellias ou de films comme, tout récemment, Bienvenue chez les Ch'tis de Danny Boon.

Enfin, on peut établir des séries taxinomiques des exotismes en se fondant sur le critère de l'institutionalisation (points [c] et [d] de la liste). Une citation tirée d'un récit de Konstantin Paoustovski est censée éclaircir l'usage du mot dans un dictionnaire: "Ah, les roses de Chiraz, les rossignols de Khorassan! il se moquait de l'ekzotika canonisé» "53. Ici, Paoustovski tourne en ridicule les poèmes persans d'Essénine; mais il pense - comme le pensaient avant lui Daudet ou Segalen - au danger que l'exotisme court en permanence, celui du kitsch et du stéréotype. Le terme qu'il utilise pour dire "canonisé» (kazennyj) a en russe le sens de bureaucratique, étatique: une piste intéressante pour la classification. On pourrait en effet imaginer de distinguer entre les formes "officielles" et "dissidentes", conformistes et non-conformistes de l'exotisme; prenons pour illustrer l'exotisme étatique la "fête brésilienne» à Rouen, en 1550, organisée en grande pompe, avec la participation d'Indiens et la démonstration de danses, costumes et artisanat (une sorte de préfiguration des Expositions universelles motivée par les ambitions françaises au Brésil) ${ }^{54}$. Dans le contexte russe, les festivités impériales ou soviétiques à l'occasion, par exemple, de l'incorporation de la Crimée tatare à la Russie (1783) ou de l'ouverture du Turksib, ligne ferroviaire reliant la Russie, la Turkménie et la Sibérie extrême-orientale (1933), incarnent avec éclat l'application au domaine de l'exotisme de cette «frénésie administrative» (administrativnyj vostorg) que démonçait avec tant de véhémence Saltykov-Chtchedrine.

52. A. Etkind, «Fuko i tezis vnutrennej kolonizacii: postkolonial'nyj vzgljad na sovetskoe prošloe», p. 49.

53. Slovar' Akademii nauk SSSR v 4 tt. (1984).

54. F. Affergan, Exotisme et altérité. Essai sur les fondements d'une critique de l'anthropologie, p. 103-104; F. Denis, Une Fête brésilienne célébrée à Rouen en 1550; B. PerroneMoisés, "L'Alliance normando-tupi au XVI" siècle: la célébration de Rouen". 


\section{Compagnons de route de l'exotisme}

Nous fondons notre approche, d'une part, sur l'idée de la complexité inhérente aux concepts chargés d'histoire et, d'autre part, sur l'idée que ceux-ci ne fonctionnent jamais seuls, mais à l'intérieur de réseaux conceptuels serrés. C'est l'interaction avec d'autres notions, complémentaires ou "concurrentes", qui donne tout son sens à la notion d'exotisme. Nous en dégageons cinq - orientalisme, édénisme, utopisme, primitivisme, régionalisme - en ordonnant quelque peu, à notre manière, la profusion d'éléments disponibles.

1. Orientalisme: c'est bien entendu le premier concurrent de l'exotisme, il exprime beaucoup d'idées qui y sont associées. Le mot même n'apparaît que dans les années 1820 pour qualifier (ironiquement) la théorie qui avait alors déjà perdu son actualité et qui insistait sur l'origine orientale de la culture et des langues européennes. Par la suite, on a dénommé ainsi deux phénomènes différents: les sciences académiques qui se consacrent à étudier différentes régions de l'Orient et un courant artistique qui s'intéresse à la thématique et à la stylistique orientales, d'abord dans les arts plastiques, puis dans toutes les autres formes d'art (musique, ballet, théâtre, littérature). Bien que d'apparition tardive, si l'on suit la définition de Said, le concept d'orientalisme apparaît comme plus fondamental que l'exotisme: c'est lui, fondé sur l'opération initiale de découpage du monde en Occident et en Orient, qui fournit le modèle de base pour la construction des objets exotiques.

Si l'on prend Said trop à la lettre, l'orientalisme est inventé par les savants du XIXe siècle afin d'asseoir et de justifier la violence du colonialisme européen. Au lieu de polémiquer contre cette conception, on soulignera qu'à partir d'un certain moment, l'Europe vit entre «l'Orient de Xerxès et l'Orient du Christ", selon le mot du philosophe Soloviev; la pression des Sarrasins, des Turcs continue, commence l'influence de la Bible, de Byzance, de Jérusalem. Les chrétiens de cette époque ne devaient pas plus s'adonner au sentiment de l'exotisme que les Grecs anciens. Mais les récits de pèlerinages aux lieux saints, emplis d'émerveillement, laissent un modèle en héritage pour les narrations des pays merveilleux à venir. C'est la passion pour le monde de la Bible, la volonté de trouver le témoignage de la Parole qui poussent à vouloir connaître l'Egypte, l'Ethiopie, la Palestine. Ce sont les recherches bibliques qui 
amènent Guillaume Postel au XVI ${ }^{\mathrm{e}}$ siècle, Athanase Kircher et Hiob Ludolf au XVII ${ }^{\mathrm{e}}$, à étudier les antiquités et les langues orientales: l'hébreu, l'égyptien, le copte. Lorsque F. Th. Vischer, en faisant sa synthèse de l'esthétique romantique et hégélienne, parle d'une "poétique orientaliste», il entend le style poétique de la Bible ${ }^{55}$.

L'exotisme européen apparaît alors comme une contamination de la ligne d'Hérodote par la ligne de la Bible. Les deux évoluent ensemble, leur tracé dévie sous l'influence de la Perse, de l'Inde, de l'Asie. Le trésor d'images de différents Orients s'accumule à travers les âges; il nourrit le sens de l'exotisme européen. Outre les liens de ce dernier avec le colonialisme, il faudrait sans doute tenir compte de ce bagage. C'est surtout vrai pour la Russie, qu'un orientalisme très particulier, conditionné en même temps par Byzance et par la Steppe asiatique, accompagne depuis sa naissance, pour laquelle il a souvent été un moyen de lutte contre l'Occident et l'occidentalisation, depuis Catherine II avec son "projet grec» jusqu'aux projets néo-eurasiens plus récents, et qui n’a pas encore été décrit dans toute sa complexité ${ }^{56}$.

2. Edénisme: ce terme chez Charles Fourier signifie l'état de l'humanité avant sa chute dans la sauvagerie; nous l'utilisons dans le sens de cette quête des îles bienheureuses ou du Paradis sur terre qui laisse sur l'imaginaire européen une empreinte des plus profondes. Il y a dans cette quête un aspect mystique et onirique; avec plus de raisons encore que les pèlerinages, les visions médiévales du Paradis peuvent être vues comme autant de descriptions proto-exotiques. Mais il y a aussi des entreprises tout à fait réelles. Michel Faucheux affirme qu'entre 1526 et 1721 quatre grandes expéditions maritimes ont été organisées pour retrouver le Paradis terrestre en suivant le fameux récit de Saint Brendan datant du VI siècle $^{57}$. Jean Delumeau insiste sur le rôle des voyages à la recherche du Paradis, dont celui de Saint Brendan, dans la conception et l'exécution de grands voyages de découverte, plus particulièrement celui

55. F. T. Vischer, Über das Erhabene und Komische: ein Beitrag zu der Philosophie des Schönen, S. 64.

56. Cf. dans la bibliographie les travaux de Goršenina, Schimmelpenninck van der Oye, Abensour, Lanne, Heller.

57. M. Faucheux, Les Quêtes chimériques. Mythes et symboles de l'Eldorado à l'amour éternel, p. 20-25. 
de Colomb ${ }^{58}$. Voilà un facteur de l'élan vers les pays exotiques que la catégorie du colonialisme ne subsume pas directement.

Soulignons que la formule édénique fonctionne conjointement avec son pendant, la formule infernale: on parlera de l'enfer vert de la jungle, de l'enfer blanc de l'Arctique, on comparera les autochtones à des démons rouges, noirs, jaunes, on verra les serviteurs de Satan en Sarrasins et en Turcs, la beauté des paysages et la simplicité des mœurs exotiques seront démasquées comme autant d'illusions sataniques. Les esprits libertins succombent à la tentation: les figures exotiques et diaboliques se chevauchent chez un Byron ou un Lermontov.

3. Utopisme: une évidence. Un seul exemple: pendant cinq cents ans, entre le XII e et le XVII e siècles, l'Europe croyait à l'existence du royaume chrétien lointain (indien? mongol? abyssinien ?) du Prêtre Jean, croyance alimentée entre autres par une lettre de ce roi adressée à l'empereur de Byzance Manuel Comnène ${ }^{59}$. Le pays qu'elle décrit, rempli de richesses, habité par des animaux merveilleux et des êtres monstrueux sortis des récits d'Hérodote, n'est-il pas exotique par excellence? Cette lettre a été lue avec passion en Russie; elle fait naître une légende comparable à celles du royaume d'Opon (Japon, Cipangu de Marco Polo), de la féerique Daourie, du pays des Eaux-Blanches (Belovod'e), ces contrées de la liberté et du bonheur que racontent les utopies populaires russes depuis le XVII ${ }^{\text {e }}$ iècle ${ }^{60}$. Par contraste avec l'exotisme de l'Etat que nous avons discuté plus haut, ne faudrait-il pas introduire la notion d'exotisme populaire? Tout en se rappelant bien entendu que l'exotisme est lié tout autant à l'utopisme savant: Thomas More compose son livre en lisant les journaux d'Amerigo Vespucci, Diderot et Bernardin de Saint-Pierre donnent à leurs traités utopiques la forme de récits des îles lointaines.

4. Primitivisme. Ne le confondons pas avec l'exotisme: les fastes orientaux n'ont rien de primitif. Arthur A. Lovejoy et George Boas expliquent, en 1935, la relation entre les deux: «l'amour de l'étrange et la révolte contre le familier", composantes du primitivisme, sont en même temps le fondement de l'exotisme. Quant au primitivisme,

58. J. Délumeau, Une Histoire du Paradis, p. 145.

59. Cf. par exemple I. Bejczy, La Lettre du Prêtre Jean, une utopie médiévale.

6o. Cf. dans la bibliographie les travaux de Čistov, Klibanov, Khokhlov, ainsi que Heller et Niqueux. 
Lovejoy et Boas en voient deux types: chronologique (qui renvoie dans le passé l'Age d'or de l'humanité) et culturel (qui considère que la vie simple est meilleure que la vie compliquée) ${ }^{61}$.

N'entrons pas dans les détails de ce débat essentiel; qu'il suffise de dire que la notion de primitivisme devient extrêmement populaire pendant le demi-siècle qui s'écoule entre la sortie de Primitive Culture (1871) d'Edward B. Tylor et celles de La Mentalité primitive (1922) de Lévy-Bruhl et de Primitive Art (1927) de Franz Boas. Les sciences humaines sont bouleversées et les arts encore plus, par l'attitude primitiviste: c'est là un des leviers qui permettent de renverser le réalisme et, avec lui, toute la tradition du mimétisme illusioniste ${ }^{62}$. Une attitude qui se décline en plusieurs versions (se superposant à la division opérée par Lovejoy et Boas) : art premier ou anté-historique (retour à la magie, au naturel), sauvage (intensité, puissance, vitalité), primitif (vision élémentaire, minimaliste), ancien (retour à l'harmonie, noble simplicité comme antidote à la dégradation causée par la civilisation technologique urbaine), enfantin (spontanéité, naïveté virginale), populaire (sagesse et authenticité), etc. Après Gauguin, il n'y a pas de courant d'avant-garde, dans tous les arts y compris la littérature, qui aurait résisté à l'attrait du "néo-primitivisme» dans l'une ou l'autre de ses variantes.

5. Régionalismes. Là encore, nous ne faisons qu'indiquer un sujet bien touffu. L'exotisme a beaucoup d'incarnations «régionalistes»: arabisme, turquisme, japonisme, etc. qui sont autant de «référents flottants» du terme. En général, ce sont des dénominations de courants ou d'autres formations idéologiques, politiques, artistiques (cf. le scythisme en Russie). Le caractère de l'exotisme dépend de son interaction avec l'aire culturelle ciblée (peut-être, aussi, avec l'aire de départ). Mais le sens de l'exotique n'est pas une somme des exotismes régionalistes concrétisés, il se situe à la fois au-delà et à l'intérieur d'eux. Ajoutons que si la «couleur locale» est essentielle pour le régionalisme, proche en cela de la position ethnographique, l'exotisme fait sien le "principe de Tartarin»: l'important pour lui est l'ambiance, une sorte d'aura qui permet autant de cacher que de montrer.

6I. A. O. Lovejoy, G. Boas, Primitivism and Related Ideas in Antiquity, p. 8.

62. Cf. P. Dagen, Le Peintre, le poète, le sauvage: les voies du primitivisme dans l'art français; J. Flam, M. Deutsch (ed.), Primitivism and XXth-century art: a documentary history; E. Barkan, R. Bush (ed.), Prehistories of the future: the primitivist project and the culture of modernism. 
Arrêtons-nous ici pour remarquer que nos notions définissent, deux par deux, trois axes: l'axe de la géographie et des aires culturelles (orientalisme/régionalisme); l'axe de l'idéologie et du temps passéfutur (édénisme/ utopisme); l'axe de l'anthropologie et de la poétique (exotisme/ primitivisme). Ce qui montre leur lien très fort, sinon leur systémicité.

\section{Pour conclure: exotismes et textes}

L'exotisme peut se manifester à tous les niveaux textuels et intertextuels, il peut contribuer à définir le cadre générique, la configuration topique ou compositionnelle, la motivologie, l'iconographie, le schéma prosodique, le symbolisme, l'aspect polygraphique de l'œuvre. L'exotique peut apparaître dans des rôles épisodiques, il peut dominer l'ensemble en tant que principe poétique. Il faut savoir différencier ses échelles, ses fonctions, sa nature. Nous proposons de faire une distinction, qui nous semble utile pour l'analyse de texte, entre les usages mythopoétique, poétique et rhétorique de l'exotisme. Le premier concerne la base esthético-philosophique d'une "exotisation du monde" particulière (les mécanismes énumérés plus haut contribuent à former cette base). Le deuxième usage matérialise cette forme particulière d'exotisation dans les textes; différents éléments exotiques y sont intégrés à l'aide de divers procédés compositionnels et thématiques, d'images, de personnages, etc. Le troisième usage répertorie de tels procédés et topoi, et fixe leurs significations; un système rhétorique apparaît qui dépend désormais plus de sa propre organisation interne que des qualités de l'objet représenté.

Considérons le japonisme. Le monde occidental découvre l'art japonais dans la deuxième moitié du XIX ${ }^{\mathrm{e}}$ siècle; cette découverte joue un rôle majeur dans la formation et l'évolution du modernisme. L'art japonais apprend à libérer le graphisme, il offre une vision insolite de l'érotisme et du grotesque. Les motifs japonais envahissent différents domaines. Certains d'entre eux seront utilisés comme des ornements tout faits, d'autres seront adoptés comme signes d'un nouveau langage plastique (la composition diagonale signifiera le dynamisme, le contraste des plans, une profondeur "naturelle» obtenue hors de l'artifice de la perspective), d'autres encore provoqueront l'élaboration de nouveaux 
principes esthétiques: la géométrisation des formes et l'agencement modulaire dans la peinture abstraite et dans l'architecture du XX $\mathrm{XX}^{\mathrm{e}}$ siècle doivent beaucoup au minimalisme japonais.

On voit comment s'interpénètrent les trois usages de l'exotisme. On voit également, au terme de notre parcours, que nous vivons dans un monde façonné par nos exotismes.

Nous ne sommes pas encore, croyons-nous, au bout de nos découvertes.

Leonid HeLler

Lausanne 


\section{BIBLIOGRAPHIE}

AваzA, M., "Japan as imagined by Arabs", Newsletter of the International Institute for Asian Studies, March 2002, http://www.iias.nl/nl/27/IIAS_NL27_19.pdf.

Abensour, G., "La Pisanelle de Gabriele d'Annunzio et le mirage orientaliste", Cahiers du Monde russe, 48/1 (2007).

Affergan, F., Exotisme et altérité. Essai sur les fondements d'une critique de l'anthropologie, Paris, PUF, 1987.

Auerbach, E., Mimésis. La Représentation de la réalité dans la littérature occidentale, Paris, Gallimard, 1968 (1946).

Baltrusaitis, J., Le Moyen Age fantastique. Antiquités et exotismes dans l'art gothique, Paris, Flammarion, 2000 (1981).

Barkan, E., Bush, R. (ed.), Prehistories of the future: the primitivist project and the culture of modernism, Stanford, Stanford U. Press, 1995.

Barthes, R., Mythologies, Paris, Seuil, 1957.

Bejczy, I., La Lettre du Prêtre Jean, une utopie médiévale, Paris, Imago, 2001.

Bessis, S., L'Occident et les autres. Histoire d'une suprématie, Paris, La Découverte, 2001.

BıÉLY, A., Kotik Letaiev (trad. Georges Nivat), Lausanne, L’Age d'Homme, 1973.

Boas, G., Primitivism and Related Ideas in the Middle Ages, Baltimore, Johns Hopkins U. Press, 1997.

Bogdanov, K., O krokodilah v Rossii. Očerki iz istorii zaimstvovanij $i$ èkzotizmov, Moskva, NLO, 2006.

BrIE, F., Exotismus der Sinne. Eine Studie zur Psychologie der Romantik, Heidelberg, Winter Vlg., 1920.

Buisine, A., Dodille, N., Duchet, C. (éd.), L'Exotisme. Actes du colloque de Saint-Denis de la Réunion, Paris, Didier-Erudition, 1988. 
Cario, L., Régismanset, Ch., L'Exotisme, la littérature coloniale, Paris, Mercure de France, 1911.

Cassagne, A., La Théorie de l'art pour l'art en France chez les derniers romantiques et les premiers réalistes, Paris, Hachette, 1906.

Célestin, R., From Canibals to Radicals: Figures and Limits of Exoticism, Minneapolis, U. of Minnesota Press, 1996.

Chinard, G., L'Exotisme américain dans la littérature française au XVI siècle, Paris, Hachette, 1911.

Čistov, K., Russkie narodnye social'no-utopičeskie legendy, Moskva, Nauka, 1967.

-, Russkaja narodnaja utopija (Genezis i funkcii social'no-utopičeskih legend), Sankt-Peterburg, Bulanin, 2003.

Dagen, Ph., Le Peintre, le poète, le sauvage: les voies du primitivisme dans l'art français, Paris, Flammarion, 1998.

Décoret-Ahina, A., Les Danses exotiques en France. 1880-1940, Pantin, Centre national de la danse, 2004.

Delumeau, J., Une histoire du Paradis, Paris, Fayard, 1992.

Denis, F., Une Fête brésilienne célébrée à Rouen en 1550, Paris, Techener, 1850.

Ducrey, G., Moura, J.-M. (dir.), Crise fin-de-siècle et tentation de l'exotisme, Villeneuve d'Asc, Univ. Lille III, 2002.

Efremova, E., Novyj slovar' russkogo jazyka, 2000.

ETKind, A., "Fuko i tezis vnutrennej kolonizacii : postkolonial'nyj vzgljad na sovetskoe prošloe», Novoe literaturnoe obozrenie, 49 (2001).

—, «Bremja britogo čeloveka, ili Vnutrennjaja kolonizacija Rossii », $A b$ Imperio (Kazan), 1 (2002).

Faucheux, M., Les Quêtes chimériques. Mythes et symboles de l'Eldorado à l'amour éternel, Paris, Lattès, 2006.

Fernandez-Bravo, N., "Le Mythe de l'Atlantide dans le romantisme allemand", in Foucrier, Ch., Guillard, L. (éd.), Atlantides imaginaires. Réécritures d'un mythe (Colloque Cerisy), Paris, Michel Houdiard, 2004.

Flam, J., Deutsch, M. (ed.), Primitivism and XXth-century art: a documentary history, Berkeley, U. of California Press, 2003.

Gide, A., Journal. 1889-1939, Paris, Gallimard (Pléiade), 1951. 
Gjujo, Ž.-M., Iskusstvo s točki zrenija sociologii, Moskva, Panteleev, 1891.

Goncourt, E. et J., Journal. Mémoires de la vie littéraire, Monaco, 1957.

Goršenina, S., "Mnogolikij Vostok russkogo hudožestvennogo orientalizma (XVIII- načalo XX veka)», in Kul'turnye cennosti. Cultural Values: 2004-2006, Central Asia in Past and Present, Sankt-Peterburg, Université de Saint-Pétersbourg, 2008.

Grin, A., "Klubnyj arap» (1918), in Sobranie sočinenij, t.5, Moskva, Pravda, 1980.

Guyau, J.-M., L'Art au point de vue sociologique, Paris, Ed. de SaintCloud, 1923.

Halen, P., Moura, J.-M., La Littérature des lointains. Histoire de l'exotisme européen au XX $X^{e}$ siècle, Paris, Honoré Champion, 1998.

Hartog, F., Le Miroir d'Hérodote, Paris, Gallimard, 2001 (1980).

—, Anciens, modernes, sauvages, [Paris], Gallade-Seuil, 2005.

Hassoun, J.-P., Raulin, A., "Homo exoticus", in Mille et une bouches. Cuisine et identités culturelles, S. Bessis (dir.), Paris, Autrement, 1995.

Heller, L., "The Russian orientalism: an encounter with the West», in Russia and the West: Missed Opportunities, Unfulfilled Dialogues, E. Waegemans (ed.), Bruxelles, Contactforum-Universa Press, 2006.

—, "L'Orientalisme russe: parenté, altérité, étrangeté?", in L'Altérité. Etudes sur la pensée russe, F. Lesourd (éd.), Lyon, Univ. JeanMoulin Lyon III, 2007.

—, "Un Aspect du nouvel orientalisme russe: le japonisme», Chroniques slaves (Grenoble), 3 (2007).

Heller, L., Niqueux, M., Histoire de l'utopie en Russie, Paris, PUF, 1995.

Il'f, I., Petrov, E., Zolotoj telenok, Moskva, Sov. pisatel', 1967.

Jasenskij, B., Čelovek menjaet kožu, Moskva, Sovet. literatura, 1933.

Jost, F., "Exotisme», Dictionaire international des termes littéraires, http://www.ditl.info/arttest/art1658.php.

Khoкhlov, G., Le Voyage de trois Cosaques de l'Oural au Royaume des Eaux Blanches, trad. M. Niqueux, Paris, L'Inventaire, 1996. 
Klibanov, A., Narodnaja social'naja utopija. Period feodalizma, Moskva, Nauka, 1977.

—, Narodnaja social'naja utopija. XIX vek, Moskva, Nauka, 1978.

Kristeva, J., Etrangers à nous-mêmes, Paris, Gallimard-Folio, 2007 (1988).

Lanne, J.-C., "Le Futurisme russe et l'art d'avant-garde japonais", Cahiers du Monde russe et soviétique, 25/4 (1984).

—, «V. Khlebnikov et l'Orient», Slavica Occitania, 8 (1999).

Layton, S., Russian Literature and Empire. Conquest of the Caucasus from Pushkin to Tolstoy, Cambridge, Cambridge U. Press, 1994.

LÉvinas, E., "Visage et violence première (Phénoménologie de l'éthique). Interview", in La Différence comme non-indifférence. Ethique et altérité chez Emmanuel Lévinas, A. Münster (dir.), Paris, Kimé, 1995.

LÉvi-Strauss, C., "Trois humanismes», in Anthropologie structurale 2, Paris, Plon, 1973.

Looff, F. W., Allgemeines Fremdwörterbuch, Langensalza, Verlag H. Beyer, 1883.

Lovejoy, A. O., Boas, G., Primitivism and Related Ideas in Antiquity, vol. 1, New York, Octagon Books, 1965 (1935).

Markov, V., Iskusstvo negrov, Petrograd, Narkompros, 1919.

Martino, P., L'Orient dans la littérature française au XVIe et au XVIII ${ }^{e}$ siècles, Paris, Hachette, 1906.

Moura, J.-M., Lire l'exotisme, Paris, Dunod, 1992.

Noa-Noa. Putešestvie na Taiti, [Moskva], Makovskij, 1914.

Perrone-Moisés, B., "L'Alliance normando-tupi au XVI e siècle: la célébration de Rouen ", Journal de la Société des Américanistes, 94-1 (2008).

Pochat, G., Der Exotismus während des Mittelalters und der Renaissance. Voraussetzungen, Entwiklung und Wandel eines bildnerischen Vokabulars, Stockholm, Almqvist-Wiksell, 1970.

Rincón, C., "Exotisch/Exotismus", in Aesthetische Grundbegriffe. Historisches Wörterbuch, K. BARCK (et al.), Bd. 2, Stuttgart/ Weimar, Verlag J. B. Metzler, 2007.

Rivière, J., Le Roman d'aventure, Paris, Editions des Syrtes, 2000.

Roussillon, A., Identité et modernité. Les voyageurs égyptiens au Japon (XIX ${ }^{e}-X X^{e}$ siècles), Arles, Actes Sud, 2005. 
Samutina, N., "Kul'tovoe kino: daže zritel' imeet pravo na svobodu", Logos, (2003).

Schimmelpenninck van der Oye, D., Toward the rising sun: Russian ideologies of empire and the path to war with Japan, DeKalb, Ill., Northern Illinois U. Press, 2001.

—, "Orientalizm - delo tonkoe», Ab Imperio (Kazan), 1 (2002).

Schulz, H. et Basler, O., Deutsches Fremdwörterbuch, Bd.5, Berlin/ New York, Walter de Gruyter, 2007.

Segalen, V., Essai sur l'exotisme, une esthétique du divers. Notes (présenté et annoté par Lelong, D.), Montpellier, Editions Fata Morgana, 1978.

Thompson, E., Imperial Knowledge. Russian Literature and Colonialism, Westport, Greenwood Pres, 2000.

Todorov, T., Nous et les autres. La Réflexion française sur la diversité humaine, Paris, Seuil, 1992.

Vernant, J.-P., Entre mythe et politique, Paris, Seuil, 2003.

Vidal-NaCQuet, P., Le Chasseur noir. Formes de pensée et formes de société dans le monde grec, Paris, La Découverte, 2005.

Vischer, F. T., Über das Erhabene und Komische: ein Beitrag zu der Philosophie des Schönen, Stuttgart, Imle u. Krauss, 1837.

Vol'demar Matvej $i$ «Sojuz molodeži», Moskva, Nauka, 2005.

Zimmer, W., "Voyages africains de recherche et de découverte à l'intérieur de l'Allemagne. L'Exotisme à rebours", in Buisine, A., Dodille, N., Duchet, C., éd., L'Exotisme. Actes du colloque de Saint-Denis de la Réunion, Paris, Didier-Erudition, 1988. 
